\title{
Présentation générale : techno-économie des risques industriels
}

\section{Sophie Boutillier ${ }^{1}$}

${ }^{1}$ Université du Littoral, CLERSE UMR CNRS 8019, sophie.boutillier@univ-littoral.fr

RÉSUMÉ. Ce numéro de Technologie et innovation regroupe six articles qui font à la fois état de l'évolution des techniques et des risques industriels, mais qui se focalisent aussi sur des activités particulières : les accidents de travail, la construction et l'urbanisation, la production d'alumine, la maintenance dans un bâtiment public et la maintenance industrielle.

ABSTRACT. This issue of Technology and Innovation consists of six papers that as well as describing general aspects of technical evolutions and industrial risks, focus on specific activities: workplace injuries, construction and urbanization, alumina production, maintenance in a public building and industrial maintenance.

MOTS-CLÉS. techno-économie, risques industriels, accidents du travail, maintenance industrielle.

KEYWORDS. techno-economics, industrial risks, workplace injuries, industrial maintenance.

Le risque technologique est constitutif de l'activité humaine, comme l'attestent les travaux des archéologues et des historiens (BONNEIL, FRESSOZ, 2016; FRESSOZ, 2012 ; MASSARDGUIBAUD, 2010). L'histoire de l'humanité est balisée par des catastrophes de toute nature, certaines sont naturelles (tremblements de terre, innovations, éruptions volcaniques, etc.), mais beaucoup d'autres sont le résultat de la créativité humaine. Les économistes invoqueront à ce propos les externalités négatives. En ce sens la connaissance a cette double identité, à la fois externalité positive en participant à l'amélioration des conditions de vie des individus et participant au développement du progrès technique, mais aussi une externalité négative, par les dégâts et catastrophes dont elle est le produit. Cette idée est largement rependue dans les représentations collectives. Dans les bandes dessinées ou les dessins humoristiques combien de fois un savant fou ne voit-il pas explosé au visage la solution soi-disant miraculeuse qu'il avait préparée ? Dans un essai très célèbre, publié en 2005, Effondrement, comment les sociétés décident de leur disparition ou de leur survie, Jared Diamond remet implicitement en question l'idée d'une fatalité face à l'événement destructeur. Si depuis Descartes, l'homme doit se rendre maître et possesseur de la nature, c'est avec difficulté qu'il y parvient, car comme le soulignait Engels (1883), dans son célèbre essai, La dialectique de la nature, la nature se venge toujours.

Ce numéro de Technologie et innovation regroupe six articles qui font à la fois état de l'évolution des techniques et des risques industriels, mais qui se focalisent aussi sur des activités particulières : les accidents de travail, la construction et l'urbanisation, la production d'alumine, la maintenance dans un bâtiment public et la maintenance industrielle.

L'article de Smail Aït-El-Hadj positionne le sujet des risques industriels dans sa dimension historique, en liaison avec l'histoire des techniques et de l'industrie. Pour ce faire, il se positionne par rapport à la théorie évolutionniste et cherche à montrer la relativité des risques industriels par rapport aux trois systèmes technologiques qui se sont succédé durant l'histoire industrielle. A des risques touchant principalement les industries minières et les machines à vapeur dans le premier système technologique, risques limités au site et au personnel, succède dans le deuxième système technologique les grands accidents chimiques et nucléaires, formalisés sous le concept de risque technologique majeur. Les risques industriels du troisième système technologique, sont marqués par leur échelle et généré par leur complexité tel que le risque cybernétique, et aussi par l'accumulation historique des nuisances de toute l'histoire industrielle pour le risque environnemental global. Cette dernière 
configuration modifie l'influence du risque industriel sur la trajectoire même de l'évolution technologique. Au fur et à mesure du développement des technologies, les risques industriels ont des conséquences à la fois de plus en fortes dans le temps (la radioactivité) et dans l'espace (les pollutions se diffusent au niveau mondial).

Dans le même esprit, Stéphane Callens étudie la question du risque industriel dans sa dimension internationale en concentrant son propos sur les accidents du travail en lien avec les risques industriels. Il part du constat que les théories de la globalisation émergent au moment d'une augmentation des accidents industriels au milieu des années 1980, mais qu'elles conjuguent le risque de manière différente : comme une «manne » apportée par la globalisation, comme une probabilité ubiquiste d'un désastre, ou encore une injustice atteignant de façon répétée le plus vulnérable. Mais, l'idée selon laquelle il existerait un lien entre globalisation liée aux nouvelles technologies et risques industriels ne va pas forcément de soi. A partir de Hall et Soskice (2002), Stéphane Callens établit une typologie des relations industrielles dans le monde, en distinguant, la «Liberal Market economy» et la « Coordinated market economy» en fonction du degré de liberté syndicale. L'étude empirique des données mondiales sur la période 1983-2014 indique quelques évolutions positives dans la période récente, ce qui est un argument en faveur de l'approche ubiquiste proposée par Ulrich Beck (2001). Ainsi, si aujourd'hui les pratiques organisationnelles étaient toutes équivalentes à celles d'un pays CME comme la Suède, il y aurait 14 fois moins d'accidents du travail dans le monde.

Jean-Claude Blesius concentre son propos sur les risques industriels liés à la construction et à l'urbanisation. Son propos se focalise tout particulièrement sur les espaces où les industries à risque cohabitent parfois de manière étroite avec des lieux de résidence. Le risque réside dans la probabilité que survienne une explosion, une fuite toxique ou un incendie. L'objectif de l'auteur est de mener une réflexion sur la construction, complexe, de la législation en matière de maitrise de l'urbanisation. Cette dernière est le résultat, entre autres, d'une volonté des pouvoirs publics de garantir un développement industriel efficace, ainsi que du retour d'expérience des catastrophes industrielles qui se sont produites au cours de l'histoire. Le Plan de prévention des risques technologiques (PPRT) impose notamment aux riverains d'installations à risque d'effectuer des travaux de sécurisation dans leurs logements. Bien que leur prise en charge financière ait fait l'objet de nombreux débats, cette disposition témoigne de l'aboutissement d'un processus qui conduit à envisager ces territoires comme un tout. Les premiers résultats de cette recherche tendent à montrer qu'aujourd'hui, les différents maillons ne se tournent plus le dos et se greffent ensemble pour former une chaîne au service du "vivre avec » le risque industriel.

Philippe Mioche s'interroge sur l'avenir de l'usine d'alumine de Gardanne, dans le sud de la France, qui est la plus ancienne du monde dans cette industrie, puisque sa construction remonte à la fin du XIX ${ }^{\mathrm{e}}$ siècle. Sa localisation est le résultat de la combinaison de trois facteurs principaux : matières premières (présence de lignite), nœud de circulation entre Marseille et Lyon, et enfin main-d'œuvre miséreuse venir d'Italie. Actuellement, cette usine toujours très polluante est à l'origine d'une controverse sur le maintien ou l'arrêt de son activité. La question est importante, car ses impacts sur la santé et l'environnement sont importants, elle s'inscrit aussi dans un processus de désindustrialisation de l'Europe. L'objectif de cet article est de retracer les grandes phases de l'histoire de cet établissement pour discuter de l'histoire des risques industriels. La fermeture de cet établissement n'arrêtera pas la production d'alumine dans le monde, l'activité sera ainsi délocalisée vers les pays en développement, moins attentifs à ces questions environnementales. Aussi le véritable enjeu réside-t-il, selon l'auteur, dans le développement de nouvelles technologies plus respectueuses de l'environnement pour conserver cette activité dans le sud de la France.

Claude Fournier étudie de façon approfondie la question de la maintenance d'un célèbre bâtiment industriel, la Cité des sciences et de l'industrie à Paris, qui se trouve au nord-est de la capitale 
française. Ce lieu a pendant près de 100 ans abrité les abattoirs de La Villette. Leur déménagement dans le sud de la région parisienne à Rungis. La décision est prise en 1960, après maintes hésitations et jeux de pouvoir politique. Mais, par la suite se pose la question de l'utilisation des lieux laissés vacants par les abattoirs, pour aboutir à l'ouverture de la Cité des sciences et de l'industrie à la fin des années 1980, dont l'objectif est de participer à la vulgarisation de la culture technique et industrielle auprès des jeunes générations. Claude Fournier pose dans son texte la question des réalisations spectaculaires destinées à recevoir un grand nombre de visiteurs, comme la Cité des sciences et de l'industrie, qui ne sont viables que parce que leurs composantes techniques, souvent très élaborées sont sûres. Le but de l'auteur est de présenter une face méconnue de la genèse de ce beau projet, ce qu'on appelle parfois de façon imagée «le travail des soutiers ». L'auteur montre la manière dont a été traité la sûreté des personnes et des biens au travers d'une politique de maintenance raisonnée en considérant que cet ensemble tertiaire, qui devait recevoir des millions de personnes, était en fait un objet industriel à qui on devait appliquer les dernières pratiques, en totale rupture avec la culture ambiante de l'époque.

Claude Pichot se focalise sur la question de la maintenance industrielle, qui souvent délaissée en raison de son coût immédiat sur la réalisation d'une construction, quelle que soit sa nature. Les autorités publiques et les industriels tendent à minorer les risques industriels, qui pourtant sont bien réels, comme en atteste l'histoire industrielle que nous avons déjà évoquée. Le déni de la possibilité des accidents dans le domaine industriel et de leurs conséquences catastrophiques reste pourtant le paradigme de notre modèle économique. Il résulte de la vision à très court terme de l'économie qui n'a d'attention que pour les cours de la Bourse, alors que les installations industrielles vivent et se dégradent au rythme découlant des lois de la physique sur lesquelles sont construites les technologies. Il existe une opposition théorique entre le profit à court terme et l'indispensable prise en compte du long terme pour éviter les accidents et les catastrophes. L'auteur revient sur l'histoire d'accidents industriels récents, comme l'accident de Fukushima, au Japon ou l'usine d'engrais chimiques AZF en France. L'accident de Fukushima s'est produit, alors qu'une série d'accidents que personne ne pensait pouvoir se produire en même temps s'est effectivement produite. Une vague de plus de 10 mètres de haut était considérée comme un maximum, oubliant qu'en 1923 une vague de 20 mètres avant déjà submergée la région. Or la maintenance industrielle permet de réduire les coûts dans le long terme et aussi d'éviter des victimes. La question se pose avec d'autant plus d'acuité dans le contexte actuel de réchauffement climatique.

\section{Bibliographie}

BONNEIL C., FreSsOz J.-B, L'événement anthropocène, Points-histoire, Paris, 2016.

DiAMOND J., Effondrement, comment les sociétés décident de leur disparition ou de leur survie, Gallimard, Paris, 2005.

ENGELS F., 1883, La dialectique de la nature, http://classiques.uqac.ca/classiques/Engels_friedrich/dialectique/engels_dialectique_nature.pdf.

FRESSOZ J.-B, L'apocalypse joyeuse. Une histoire du risque technologique, Seuil, Paris, 2012.

Hall P. A., SoskiCE D., Les variétés de capitalisme, L'année de la régulation, no. 6, p. 47-124, 2002.

MASSARD-GuIBAUd G., Histoire de la pollution, France 1789-1914, Editions de 1'Ecole des Hautes études en sciences sociales, Paris, 2010. 